\title{
Montage et résistance du réel chez S. Kracauer : Photographie, cinéma, texte
}

\author{
Par MAUD HAGELSTEIN \& JEREMY HAMERS
}

FNRS - Université de Liège ; Université de Liège

\begin{abstract}
C'est un peu comme si, historiquement parlant, les tranchées ouvertes dans l'Europe de la Grande Guerre avaient suscité, dans le domaine esthétique comme dans celui des sciences humaines [...], la décision de montrer par montages, c'est-àdire par dislocations et recompositions de toute chose. Le montage serait une méthode de connaissance et une procédure formelle nées de la guerre, prenant acte du «désordre du monde ». Il signerait notre perception du temps depuis les conflits du $X X^{e}$ siècle : il serait devenu la méthode moderne par excellence ${ }^{l}$.
\end{abstract}

\section{Montage déréalisant $v s$ principe de réalité}

En tant que témoin et acteur des vifs débats sur le montage qui marquent l'Europe artistique et intellectuelle de l'après-guerre, Kracauer rédige dans les années 1920 une série de "feuilletons » sur la modernité weimarienne repris dans L'Ornement de la masse (1963). Un texte en particulier retiendra ici notre attention qui permet de poser, en dehors des analyses vouées au cinéma, la question du montage. Ce texte, «Le Hall d'hôtel», est extrait d'un ouvrage sur le roman policier non publié à l'époque. Il vise à énoncer le problème du sens dans une société où la religion s'effondre progressivement. Selon Kracauer, la production artistique est toute entière prise dans cette vocation de solutionner la perte de sens consécutive à la modernité. Comme

${ }^{1}$ Georges Didi-Huberman, Quand les images prennent position. L'Eil de l'histoire (tome 1), Paris, Éditions de Minuit, 2009, p. 86. 
il l'évoque en des termes apparemment naïfs, plus le monde se déréalise, plus l'art doit exercer son rôle d'éducateur ${ }^{1}$. Là où le monde est devenu muet, là où son sens général s'est obscurci, la forme esthétique peut encore produire selon Kracauer une « sorte de langage ». L'art permet en effet d'exprimer à nouveaux frais les relations existant entre des éléments décousus et « reposant les uns à côté des autres en ordre dispersé ». Vecteur d'une sortie de l'état désenchanté du monde moderne, l'éducateur se définit par conséquent chez Kracauer comme celui qui relie entre eux des éléments épars. Et l'artiste devient bien un tel éducateur lorsqu'il vise, à travers un «organisme esthétique », une totalité qui permet de ressaisir les " éléments d'un monde en décomposition dispersés à l'aveuglette $»^{2}$. Or, dans ce texte de jeunesse déjà, Kracauer introduit une nuance : car si le rôle de l'artiste est déterminant dans sa tentative de résoudre le désenchantement du monde, il risque aussi de s'engager sur la voie néfaste qui consisterait à ré-enchanter le monde de manière abusive. Face à ces éléments dispersés qu'il s'agit de réorganiser, l'artiste peut soit être un véritable éducateur-voyant et restituer avec justesse les liens entre les éléments (il est alors du côté du «bon» montage qui respecte la réalité), soit construire un monde totalement artificiel, potentiellement enclin à donner une idée fausse de la réalité, voire idéologiquement trompeuse, en somme une sorte de «mauvais » montage. Kracauer se méfiera jusqu'à la fin de sa vie de ce montage-là, sans cependant jamais définir précisément le "principe de réalité » auquel s'adosse la normativité établie par lui comme fondement de toute l'esthétique matérielle. Et ses propos manifestent bien la complexité du problème :

L'unité de la création esthétique, sa manière de répartir les accents et de lier les événements, amène le monde qui ne dit rien à parler, confère du sens aux thèmes qui y sont abordés ; ce qu'ils signifient respectivement reste bien sûr à interpréter et dépend principalement du degré de réalité de leur créateur ${ }^{3}$.

Si l'on s'en tient à la définition de l'artiste-éducateur ayant le pouvoir spécifique de restituer au monde fracturé ou désenchanté une image unifiée et cohérente, on doit dès lors considérer le réalisateur fasciste - pour prendre un exemple extrême - comme quelqu'un dont les œuvres sont frappées de

${ }^{1}$ On verra par la suite que dans sa Théorie du film (1960) Kracauer revient implicitement sur ces propositions.

${ }^{2}$ Siegfried Kracauer, «Le Hall d'hôtel », dans Id., L'Ornement de la masse. Essai sur la modernité weimarienne, trad. fr. S. Cornille, Paris, Éditions La Découverte, 2008, p. 149.

${ }^{3}$ Ibid., p. 148. 
réussite : en effet, celui-ci est un excellent monteur qui exploite les potentialités de la situation (un monde en ruines) pour livrer aux masses une image lisse et rassurante du réel. Dans De Caligari à Hitler, la perte de contact avec l'empirie constitue déjà le problème essentiel à partir duquel s'échafaude la critique du film de propagande. Kracauer montre en effet dans ses analyses que le régime nazi travaille à étouffer la réalité, à recomposer le réel en brouillant la perception des contradictions sociales, et à transformer le réel en spectacle pour lui donner un caractère d'extériorité. Le Reich devient une œuvre d'art totale, s'appuyant sur l'appétit visuel du public et produisant un «déluge d'images» par lequel s'édifie une autre réalité, une "pseudoréalité » utopique s'adressant directement aux sens. Voilà ce qui justifie la suspicion de Kracauer à l'égard du montage et de ses effets potentiellement déréalisants ${ }^{1}$. Pour se prémunir des effets d'illusion, l'esthétique matérielle se donne pour consigne, a contrario, de « respecter au mieux la réalité ». Plus précisément, l'idée d'un médium cinématographique dont la propriété principale serait d'enregistrer le réel, sert chez Kracauer de garde-fou et le pousse à se méfier de toutes les mises en scène, de toutes les fausses reconstructions, de tous les montages biaisés qui nous en éloignent. Cette attitude amène apparemment l'auteur - de manière parfois paradoxale — à se méfier de toute une série de productions cinématographiques, ou en tout cas à identifier une veine de films susceptibles de faire basculer le spectateur du côté de l'illusion: le cinéma de Méliès et ses artifices ingénieux, par exemple, ou de manière générale " la plupart des films expérimentaux » ou «construits sur le mode de la narration théâtrale». Même les films documentaires prennent le risque de verser dans l'illusion abusive lorsque « des plans de la vie réelle ne servent qu'à illustrer un commentaire oral qui se suffit à lui-même $»^{2}$. L'artiste doit donc toujours rester vigilant afin de ne pas perdre le contact avec la réalité empirique.

\footnotetext{
${ }^{1}$ On voit bien ce qui pose problème à Kracauer. Pour rappel, il rédige sa Théorie $d u$ film après avoir travaillé pendant des années au projet d'une histoire psychologique et critique du cinéma allemand : De Caligari à Hitler. Par conséquent, la Théorie du film de Kracauer est déterminée par ses recherches antérieures sur le cinéma allemand de la République de Weimar, d'une part, et sur les films de propagande nazis, d'autre part. Il s'agit donc d'une " esthétique d'après Auschwitz » comme les commentateurs l'ont souvent rappelé.

${ }^{2}$ Siegfried Kracauer, Théorie du film. La Rédemption de la réalité matérielle, trad. fr. D. Blanchard et C. Orsoni, Paris, Éditions Flammarion, 2010, p. 73. Version originale : Siegfried Kracauer, Theory of Film. The Redemption of Physical Reality, New York, Oxford University Press, 1960.
} 
Partant de cette proposition qui reste, à certains égards, vague, on tentera de préciser et de problématiser l'ambivalence de Kracauer à l'égard de l'outil "montage», notamment dans le champ cinématographique, en ouvrant notre réflexion par cette double question: comment définir un « mauvais usage » du montage et, à l'opposé, comment peuvent se mettre en place les conditions d'un "bon usage » du montage selon Kracauer? Pour y répondre, on cherchera d'abord, dans l'œuvre de Kracauer, les éléments explicites et implicites - puisque l'on abordera aussi la pratique kracauerienne du montage textuel - qui permettront de saisir au plus près ce que serait, selon l'auteur, le principe de réalité. Ensuite, une œuvre photographique, contemporaine des quelques textes évoqués, Menschen des 20. Jahrhunderts d'August Sander, nous aidera à mieux circonscrire les possibles concrétisations de cette pensée en la mettant à l'épreuve d'une réalisation en images et en montage.

\section{La résistance du réel malgré tout}

Si le cinéma fasciste allemand occupe sans conteste la première place au rang des cinématographies soumises à la construction d'une " pseudo-réalité », les « qualités esthétiques » de l'iconographie nazie n'empêchent pas Kracauer d'identifier quelques ratés et de tirer la conclusion suivante :

Les images sont encore plus réfractaires que le commentaire. La raison en est que la réalité non montée est porteuse de sa propre signification qui parfois mine de l'intérieur la signification de propagande montée sur les prises de vues des actualités ${ }^{1}$.

Autrement dit: malgré le montage et ses effets potentiellement lissants ou totalisants, l'image conserve parfois son contenu de sens propre qui résiste de l'intérieur au projet du monteur - et en particulier dans le cas de l'image d'actualité que le film de propagande nazie n'hésite pas à s'annexer. L'image alors ne manque pas de laisser échapper des significations qui n'étaient pas voulues par le réalisateur. Selon Kracauer, bien entendu, ce qui résiste ainsi dans l'image, c'est bien la réalité elle-même : on touche alors aux limites du processus déréalisant entrepris par certains cinéastes. Autrement dit encore : là où le cinéma de propagande tentait de nier l'inertie et la résistance de la réalité, il arrive que celle-ci pointe néanmoins son nez. Dans les deux grands

${ }^{1}$ Siegfried Kracauer, De Caligari à Hitler, trad. fr. C. B. Levenson, Paris, Éditions L'Âge d'Homme, 1990, p. 351. 
films de campagne nazis analysés par Kracauer dans son texte «La Propagande et le film de guerre nazi » (Baptême du feu et Victoire à l'Ouest), de nombreuses scènes censées éblouir le spectateur le laissent en réalité dubitatif. En témoignent notamment les vues de colonnes infinies de prisonniers en marche qui, plutôt que de manifester le triomphe allemand, génèrent surtout de l'ennui : "Au lieu de se sentir accablé, le public se fatigue rapidement, ceci d'autant plus que toutes les colonnes, y compris celles des soldats allemands, se ressemblent $\gg{ }^{1}$. Un autre exemple choisi par Kracauer est encore plus éloquent. Il s'agit d'une séquence prise dans une bande d'actualités allemandes montrant Hitler accompagné d'une poignée de membres de son État-major, visitant Paris très tôt le matin : ils passent par la Concorde, les Champs-Élysées, etc., et terminent sur la terrasse du Trocadéro regardant vers la Tour Eiffel. Kracauer remarque alors : « Le Führer visite la capitale européenne conquise - mais en est-il réellement l'hôte ? Paris est aussi tranquille que grave » - devenue ville fantôme sans aucune âme pour saluer le dictateur. Tandis que Hitler inspecte Paris, "Paris lui-même ferme les yeux et s'éloigne $»^{2}$.

\section{${ }^{1}$ Idem.}

2 Idem. Plusieurs réalisateurs, essayistes et analystes des images ont fondé leur travail sur la recherche de ces lieux et moments dans lesquels le réel résiste. Dans son travail récent sur diverses représentations cinématographiques de la Shoah, Sylvie Lindeperg par exemple s'est notamment penchée sur un film célèbre dont le titre officiel est Terezin. Documentaire sur la zone de peuplement juif mais qui est entré dans l'histoire sous le titre Le Führer offre une ville aux juifs. À grand renforts d'événements mis en scène et d'effets de montage qui montrent à quel point les juifs seraient heureux dans leur camp, ce faux documentaire destiné à tromper la vigilance de la Croix Rouge Internationale, construit un monde factice. Dans La Voie des images, Lindeperg traque tous les bords de cadre, reflets, visages aux arrière-plans etc., dans lesquels on peut identifier des expressions qui contredisent les sourires joués par les prisonniers acteurs. Au-delà du cynisme qui consiste à mettre en scène des malades tout souriants dans l'hôpital de la ville, Lindeperg découvre alors plusieurs parties d'images dans lesquelles le réel filmé résiste au projet global du film. Il en va ainsi dans ce plan où l'on voit qu'une des malades dans le dortoir de l'hôpital ne regarde pas la caméra, mais lui tourne le dos, non sans tenir un miroir via lequel elle renvoie un visage inquiet et furieux à la caméra : "Ces rares gestes et regards échappant au totalitarisme de la mise en scène rapprochent certains plans du film Theresienstadt de ce que Georges Didi-Huberman désigne comme une "imagedéchirure" : derrière l'écran de la propagande fuse un "éclat de réel" qui invite à envisager la place de la caméra, à réfléchir au dispositif d'enregistrement. » (Sylvie Lindeperg, «Prise et reprise : ce qui dans l'image gît et résiste », dans Jean-Pierre Bertin-Maghit (dir.), Lorsque Clio s'empare du documentaire, vol. 2: «Archives, 
En première lecture, le réel déploie donc une forme de résistance aux intentions propagandistes et déréalisantes des documentaristes grâce à l'enregistrement d'une réalité qui peut échapper aux programmes que le réalisateur tente de lui imposer. Il ne faut pourtant pas s'y tromper. Kracauer n'attribue pas cette capacité de résistance à un hypothétique pur enregistrement du réel. Car, comme il le rappelle au sujet de la photographie, la réorganisation de ce qui a été prélevé sur le réel au profit de la construction d'une " pseudo-réalité », est déjà à l'œuvre lors du prélèvement lui-même. Il ne peut donc être question d'une pure reproduction - en miroir — de la réalité :

Les photographies ne sont pas de pures et simples copies de la nature : elles la métamorphosent en transférant sur un plan des phénomènes tridimensionnels, en tranchant leurs liens avec leurs entours, en substituant le noir, le gris et le blanc à leur gamme de couleurs ${ }^{1}$.

Techniquement, par les différentes opérations qu'elle requiert (cadrage, etc.), la photographie s'écarte inévitablement, une première fois, de la réalité. C'est peut-être même parce qu'elle s'en écarte inévitablement qu'elle doit - dans un deuxième mouvement - travailler à la mettre en valeur. Or, selon Kracauer, si la photographie s'écarte de la réalité, ce n'est pas d'abord dû au fait qu'elle cherche à la transformer, mais parce que le processus cognitif par lequel nous connaissons le réel, en lui-même, est nécessairement toujours déjà structurant. Voir c'est déjà organiser le matériau visuel et structurer le flux des impressions. À moins de prendre des photos de manière quasiautomatique et de laisser alors l'activité structurante au seul spectateur, «l'objectivité, au sens du manifeste réaliste, est hors d'atteinte ${ }^{2}$. À rebours des réalistes extrémistes qui revendiquent l'objectivité des formes artistiques, Kracauer concède donc le caractère inévitablement déterminé du regard et reconnait la détermination du dispositif d'enregistrement lui-même :

Dans ces conditions, aucune raison au monde ne saurait imposer au photographe de sacrifier ses facultés formatrices à la tentative nécessairement vaine

témoignage, mémoire », Paris, INA Éditions, L'Harmattan, 2011, p. 47. Voir aussi : Sylvie Lindeperg, La Voie des images, Lagrasse, Éditions Verdier, 2013.

${ }^{1}$ Siegfried Kracauer, Théorie du film. La Rédemption de la réalité matérielle, op. cit., p. 44.

${ }^{2}$ Rappelons ici que le Manifeste réaliste mentionné par Kracauer est un texte fondateur du Constructivisme rédigé par le sculpteur Naum Gabo et cosigné par son frère Anton Pevsner. 
d'atteindre à une telle objectivité. Dès lors qu'ils seront gouvernés par sa détermination d'enregistrer et de révéler la nature, ses choix, guidés par sa sensibilité, concernant le motif, le cadrage, l'objectif, le filtre, l'émulsion ou le grain seront parfaitement justifiés. Mieux encore, il devra faire des choix s'il veut transcender la condition minimale. Car il n'y a guère de chances que la nature se donne à lui s'il ne cherche pas à l'absorber de tout son être et de tous ses sens tendus. Dans ces conditions, la tendance formatrice n'a aucune raison de faire obstacle à la tendance au réalisme. Bien au contraire : elle peut contribuer à la concrétiser et à l'accomplir — et une telle interaction, les réalistes du XIX ${ }^{\mathrm{e}}$ siècle ne pouvaient nullement la pressentir ${ }^{1}$.

Par ailleurs, tout comme la résistance du réel n'est pas à trouver exclusivement dans l'enregistrement illusoire de la réalité, le montage n'est pas nécessairement fatal à ce réel. Il peut aussi susciter cette résistance en jouant par exemple sur l'hétérogénéité de ses composantes. Concrètement, Kracauer affirme par exemple qu'il est possible d'« intégrer à une narration des plans porteurs de significations non spécifiées $\rangle^{2}$ et de permettre à des images (des visages, entre autres) d'apparaître sans que leur signification précise pour l'histoire ne soit déterminée aussitôt. Un personnage peut ainsi surgir à différents endroits du scénario, sans que l'on sache pourquoi, avec pour effet d' « exciter notre imagination »: « Ce n'est que lors de sa réapparition qu'il revêt un rôle défini qui met automatiquement un terme à nos spéculations sur ses potentialités $\rangle^{3}$.

À suivre l'auteur, le réalisateur russe Eisenstein maîtrisait cette stratégie à merveille, et distinguait dans son matériau les images porteuses de significations dominantes et celles qu'il qualifiait (par analogie avec la musique) d' " harmoniques ». Or, selon Kracauer, cet usage des harmoniques se traduisait parfois chez Eisenstein (surtout après ses plus grands films, Octobre ou le Cuirassé Potemkine) par une certaine lourdeur, un «côté

\footnotetext{
${ }^{1}$ Siegfried Kracauer, Théorie du film. La Rédemption de la réalité matérielle, op. cit., p. 40-41.

${ }^{2}$ Ibid., p. 121.

${ }^{3}$ Idem. En parlant de «plans porteurs de significations non spécifiées », Kracauer ouvre un problème tout-à-fait déterminant. Il fait notamment référence à un article de Lucien Sève défendant la proposition suivante : le plan cinématographique offre cette particularité qu'il «ne donne pas beaucoup plus d'explications que la réalité » elle-même et parvient du coup à «délimiter sans définir » (ibid., p. 120). Mais si Kracauer parle ici d'indétermination, c'est pour limiter aussitôt la possibilité d'un flottement des significations, car elles finissent toujours, dans l'espace du film, par trouver une forme définie, et définitive.
} 
laborieux ». Par contre, son montage défendait au début une certaine souplesse :

Citons encore ces images du Cuirassé Potemkine qui n'ont qu'un lien lâche avec le déroulement de l'histoire mais qui nous imprègnent de leurs multiples significations : silhouettes de bateaux dans le port; ombre des matelots qui transportent le corps de leur camarade mort le long d'un escalier de fer, etc.

Kracauer considère ainsi certaines scènes où Eisenstein se "délecte des libertés qu'il prend avec les exigences de l'histoire » et enregistre toute une série d'impressions sensorielles sans lien direct avec la narration et ce, "sans trop se préoccuper de savoir en quoi elles contribuent à l'effet d'ensemble de l'histoire proprement dite $»^{1}$. Autrement exprimé, le vrai matériau des films d'Eisenstein - à suivre l'analyse de Kracauer — ne se réduirait pas aux «significations explicites» mais puiserait dans le «tissu d'impressions et d'expressions » de l'existant matériel.

L'exemple d'Eisenstein nous semble problématique dès lors que certains de ses films (dont ceux que cite Kracauer) jouent massivement d'inserts d'images qui, aussi éloignées soient-elles de la trame narrative principale, sont ainsi mises au service de la continuité signifiante du film ${ }^{2}$. De plus, la métaphore elle-même a de quoi étonner car les harmoniques sont en musique des vérificateurs d'unité. Elles ne font pas éclater l'ordre dominant mais le renforcent plutôt subtilement. Bien évidemment, à voir et à revoir aujourd'hui certains des films défendus par Kracauer, on ne peut que s'étonner de la distinction établie. Historiquement aussi, les maîtres russes étaient, comme on le sait, parmi les premiers inspirateurs des chefs de file d'un certain cinéma nazi, notamment celui de Leni Riefenstahl. Et on ne peut s'empêcher de voir dans la distinction implicite proposée par Kracauer un résidu de procès d'intention, gagné par les «Soviétiques », et dont Susan Sontag dénoncera les travers plus tard dans son article fameux « Fascinating Fascism $»^{3}$.

Pour dépasser l'incohérence que nous croyons identifier ici dans la lecture historique de Kracauer, il faut fusionner les deux éléments que nous avons distingués jusqu'à présent: la résistance du réel à l'intérieur d'une image et la résistance du réel suscitée par le montage. En d'autres termes:

${ }^{1}$ Ibid., p. 118.

${ }^{2}$ La forme extrême la plus directive de cet usage de deux images hétérogènes est le montage-attraction.

${ }^{3}$ Susan Sontag, «Fascinating Fascism», dans The New York Review of Books, 6 février 1975. 
complexification du réel en guise d'opposition au lissage par montage et résistance du réel à l'intérieur d'un montage lissant. Si l'on superpose ces éléments dans un même processus d'assemblage, le " bon montage » serait alors celui qui retourne à (ou qui rejoue) une complexité de la réalité tout en concédant à l'image sa capacité à résister par " excès de réalité » et sans pour autant nier l'intervention nécessaire, directe et subjective du photographe ou du réalisateur sur ce réel dont il se sert comme matière première. Ce projet n'a jamais été formulé de la sorte par Kracauer. Si l'on affranchit cette pensée du montage de son seul champ d'application visuel, on peut en revanche en trouver une expression partielle dans un agrégat de textes que Kracauer rédige et publie, d'abord en pièces détachées, dans le courant des années vingt : Les Employés.

\section{Résistance par montage}

Comme on l'a dit, l'activité du cinéaste est nécessairement structurante ou « formatrice » - pour respecter le langage de Kracauer - tant lors de la prise de vue que de l'assemblage des images. Kracauer semble suggérer toutefois qu'un usage non contraignant du montage est possible pour autant que soit respecté le principe de réalité. Il y a un équilibre à trouver, entre le montage cadenassé qui asservit le réel à un projet idéologique fort et le pur enregistrement du réel dépourvu de forme (et donc de sens).

Dans Les Employés, Kracauer propose un type d'agencement qu'il ne faut certes pas confondre avec le montage cinématographique mais dont la finalité - une «recomplexification» du monde - semble rencontrer les questions que nous adressons à la pensée du montage chez l'auteur ${ }^{1}$. Pour le dire une première fois, Les Employés réalise l'idée d'une complexification du monde par un montage que l'auteur appelle «mosaïque », pour contrecarrer une lecture lissante et homogénéisante (donc erronée) du monde. En d'autres

${ }^{1}$ Dans son introduction à l'édition française de 2004, Nia Perivolaropoulou emploie déjà cette analogie du montage cinématographique, en se basant toutefois sur les multiples aller-retours et éclairages réciproques que les articles établissent entre eux grâce à la libre circulation du lecteur à l'intérieur de l'ouvrage. À cet égard, l'analogie entre montage textuel et montage cinématographique bute sur un écueil élémentaire : soumis au flux unique de la projection, le spectateur de cinéma n'est pas en mesure de circuler librement dans le continuum du film. Nous y reviendrons plus longuement dans la suite du présent texte. Nia Perivolaropoulou, «Présentation ", dans Siegfried Kracauer, Les Employés. Aperçus de l'Allemagne nouvelle (1929), Paris, Éditions de la Maison des Sciences de l'Homme, 2004, p. 12. 
termes, et selon les vœux du sociologue, Les Employés se caractérise avant tout par un souci de retour au réel là où les scénarii que se donnent les employés (appartenance factice et illusoire à une sphère bourgeoise) sont mis en défaut par la réalité elle-même, à savoir la prolétarisation de leur catégorie socio-professionnelle à Berlin. Dans le préambule de cette mosaïque d'observations de terrain et d'entretiens, Kracauer annonce un travail composé de « citations, de conversations et d'observations directes » qui « ne sont pas là pour illustrer une quelconque théorie, mais constituent des cas exemplaires de la réalité ${ }^{1}$. Très soucieux que son ouvrage ne soit pas lu comme une somme théorique qui proposerait un ensemble d'analyses cohérentes d'une multitude de micro-cas, l'auteur place néanmoins son travail sous le signe d'une prise de conscience opératoire qui constitue, en soi, une forme d'émancipation politique :

Ce travail est un diagnostic et comme tel, il s'abstient consciemment de proposer des améliorations. Les prescriptions n'ont pas partout leur place, et ici moins qu'ailleurs, puisqu'il s'agissait avant tout de prendre la mesure d'une situation à peine entrevue jusqu'alors. De plus, si la connaissance de cette situation est une condition incontournable de toute transformation, elle représente elle-même une transformation. Car si la situation est bien connue, toute intervention sur elle dépendra de cette conscience renouvelée. Au reste, on n'aura aucun mal à trouver dans ces pages toute une série de remarques qui vont au-delà de l'analyse ${ }^{2}$.

Pour Kracauer, Les Employés lève donc un voile sur le réel ou propose, par prélèvement concrets, un retour à une réalité oubliée par les employés euxmêmes, trop attachés à leurs illusions bourgeoises et ayant, comme le dit l'auteur, « tant de mal à parler d'eux-mêmes " ${ }^{3}$. En somme, l'auteur imagine un travail de collecte de terrain, une sorte d'observation participante aussi, qui devrait permettre un retour au réel et faire œuvre, par là même, d'émancipation sociale par une prise de conscience des premiers concernés ${ }^{4}$.

Conformément aux termes du débat posé en introduction, on peut avancer que Les Employés répond à un "monde désenchanté» sans pour

\footnotetext{
${ }^{1}$ Ibid., p. 17.

${ }^{2}$ Ibid., p. 18.

${ }^{3}$ Ibid., p. 19.

${ }^{4}$ Bien entendu, le choix des employés comme catégorie sociale est primordial dans le cadre de ce projet émancipatoire étant donné que les textes qui composent l'ouvrage sont d'abord destinés à la $F A Z$, lue précisément par les employés, amenés donc à « se » lire.
} 
autant le ré-enchanter. À l'homogénéisation et au lissage, Kracauer préfère la complexification du monde et le retour au réel. Sa méthode remonte et met en tension une série d'éléments prélevés sur le réel de son temps de façon à échapper au simplisme des tableaux sociologiques habituels tout autant qu'à l'illusion dont se bercent encore les employés. L'objectif avoué de ce remontage : proposer une perspective critique sur les structures cachées de la réalité sociale. Il ne s'agit plus, comme Kracauer le suggérait dans son texte sur Le Hall d'hôtel à propos de l'art, de remettre en ordre un monde en morceaux, mais bien de complexifier la réalité en faisant éclater les versions simplistes que l'on pourrait en donner. Telle est bien la fonction critique des études micrologiques de la modernité menées par Kracauer: déjouer les « homogénéités apparentes » de l'institution, de la communauté ou du groupe social $^{1}$.

La recherche d'un équivalent cinématographique à ce type de montage est problématique a priori. L'accumulation de bribes dans une visée sociologique (qui résume bien certaines démarches qu'entreprendra notamment le Cinéma Vérité français au début des années soixante ${ }^{2}$ ) pour révéler la complexité d'un monde réduit au schéma simplificateur et préétabli, aboutit nécessairement, en raison de la longueur réduite du film, mais surtout de sa tendance à inscrire la pratique de la juxtaposition dans une dynamique de la continuité, à une forme de lissage, ne fut-ce que dans la posture spectatorielle qu'il induit, nécessairement déterminée par une linéarité temporelle dont peut s'affranchir le livre de compilation. Parce qu'il empêche la déambulation libre, parce qu'il exclut une reconfiguration perpétuelle, le film tel que l'a connu Kracauer en tant que continuum unique, apparaît a priori comme fondamentalement incompatible avec le projet d'une juxtaposition d'éléments hétérogènes qui éviterait l'imposition d'une nouvelle lecture - unique - du monde. Car le montage - non seulement peut mais — doit paradoxalement enfermer cette spontanéité qui est le propre de l'image enregis-

${ }^{1}$ Voir à ce sujet : Sabrina Loriga, « Le Mirage de l'unité historique », dans Philippe Despoix, Pierre Schöttler (eds.), Siegfried Kracauer, penseur de l'histoire, SaintNicolas-Paris, Presses de l'Université Laval-Éditions de la Maison des Sciences de l'Homme, 2006, p. $41 \mathrm{sq}$.

${ }^{2}$ La tension dialectique entre cinéma et sociologie est déjà au centre de quelques débats contemporains de l'émergence de ce mouvement documentaire. Voir notamment : Lucien Goldmann, «Chronique d'un été. Vérité scientifique, Réalisme cinématographique et Valeur esthétique », dans Cinémaction, $\mathrm{n}^{\circ}$ 17, février 1982, p. 130132. Paru d'abord en tant que : "Cinéma et sociologie. Réflexions à propos de Chronique d'un été », dans Le Cinéma et la vérité, Artsept, n², 1963. 
trée mécaniquement dans une narration, c'est-à-dire dans une trame signifiante organisée pour annihiler toute forme de hasard.

Pourtant, malgré cette contrainte liée à la forme cinématographique, l'image enregistrée - qu'elle soit photographique ou filmique - se distingue aux yeux de Kracauer par une «affinité pour le fortuit 》 précisément opposée au montage contraignant, ce qui «signifie une fois encore que le médium se prête mal à des images qui ont l'air d'avoir été emprisonnées dans "un agencement trop manifestement concerté" $»{ }^{1}$. Autrement dit: en tant qu'enregistrement du réel, l'image penche naturellement vers l'inorganisé et le diffus et ce penchant, qui est sa constitution même, ne devrait idéalement pas être effacé au profit d'une organisation trop contraignante. Cette attirance naturelle pour l'inorganisé, l'aléatoire et le mouvement doit donc paradoxalement être respectée tout en se soumettant presque inévitablement au principe du montage linéarisant (et uniformisant). Kracauer décline donc l'incompatibilité assumée entre montage et tendance à l'inorganisé, et adresse implicitement aux cinéastes une double injonction liée à deux contraintes difficilement conciliables.

D'une part, pour faire progresser une action, le cinéaste doit travailler à assigner à chaque image une signification. On peut se souvenir du principe énoncé par Panofsky : le cinéma ne peut obéir comme les autres formes artistiques à une lecture iconographique (une lecture interprétative de son sens symbolique), au sens où ce ne sont pas des idées qui s'incarnent dans une matière neutre que l'on peut modeler afin qu'elle porte ces idées, mais plutôt un matériau préexistant, déjà chargé, dont on part pour faire émerger des idées (du bas vers le haut). Cette conception de la charge symbolique des images, héritée de la philosophie cassirérienne, implique une sorte de collage potentiellement artificiel entre l'image du monde matériel et la signification qui lui est apposée. Voilà pourquoi, de temps à autre, l'entreprise narrative subit un échec. Par exemple lorsqu'une image résiste au sens qu'on lui colle, que « la rustine saute» et que l'image reprend donc sa liberté. C'est ce qui permet encore à Kracauer de penser le montage comme un « rétrécissement des significations $»^{2}$. Il en prend pour preuve l'expérience menée par le théoricien russe Koulechov avec le réalisateur Poudovkine : «Afin de prouver l'influence du montage sur la signification des plans, [Koulechov] inséra dans des contextes narratifs différents un seul et même plan du visage

${ }^{1}$ Siegfried Kracauer, Théorie du film. La Rédemption de la réalité matérielle, op. cit., p. 53.

${ }^{2}$ Ibid., p. 120. 
délibérément inexpressif de l'acteur Mosjoukine ${ }^{1}$. Un même visage pouvait donc exprimer le chagrin ou la joie en fonction du contexte signifiant qu'il intégrait.

D'autre part,

le réalisateur désire monter et explorer la réalité matérielle pour elle-même. Ce qui exige des plans qu'ils conservent la multiplicité de leurs significations, qu'ils restent capables d'exprimer leur potentiel de correspondances psychologiques. Aussi (le réalisateur) doit-il veiller à ce que le visage de Mosjoukine conserve quelque chose de son indétermination vierge ${ }^{2}$.

Autrement dit, pour éviter le rétrécissement total des significations par le montage, et afin de conserver la possibilité de correspondances multiples entre les significations (c'est-à-dire pour maintenir une certaine dynamique entre les images), le réalisateur doit ne pas recouvrir trop fermement les images de significations arrêtées. Il faut qu'un certain jeu puisse subsister.

Plutôt que d'approfondir l'injonction paradoxale assumée par Kracauer (conserver une part d'indétermination tout en s'efforçant de doter chaque image d'une signification) du point de vue des analyses de films proprement dites, on voudrait ici, pour clôturer provisoirement notre réflexion, revenir à un travail photographique partiellement contemporain du livre de Kracauer sur Les Employés, et dans lequel on peut trouver une première réalisation en images d'un art « kracauerien » du montage.

\section{Menschen des 20. Jahrhunderts}

Dans Menschen des 20. Jahrhunderts, une galerie de portraits d'allemands identifiés en fonction de leur profession, August Sander juxtapose une série de « cas exemplaires » qui rendent à l'Allemagne une certaine multiplicité si pas complexité que les nazis notamment, quelques années plus tard, ne voudront voir. L'analogie avec Les Employés paraît évidente. Cette galerie de portraits que Sander met plus de trente années à réaliser mais dont il publie une première version à la fin des années 1920, entend, comme le précise Alfred Döblin (un autre « chroniqueur» fameux de la République de

${ }^{1}$ August Sander, Antlitz der Zeit. Sechzig Aufnahmen deutscher Menschen des 20. Jahrhunderts, Munich, 1929.

${ }^{2}$ Siegfried Kracauer, Théorie du film. La Rédemption de la réalité matérielle, op. cit., p. 120. 
Weimar) dans la préface au recueil publié en $1929^{1}$, établir une sorte de sociologie en images :

On a devant soi une sorte d'histoire culturelle, mieux, une sociologie [...]. Comme on écrit la sociologie sans écrire, c'est à dire en donnant plutôt des images, des images de visages et non de costumes traditionnels ; c'est ce que réussit le regard du photographe [August Sander], son esprit, ses observations, son savoir et avant tout son immense savoir-faire photographique ${ }^{2}$.

Au centre du projet de Sander se trouve l'idée d'une collecte de manifestations exemplaires qui pourraient tenir tête à l'idée stéréotypée que l'on se fait à l'époque de certaines catégories sociales. Ces images sont rassemblées dans un ouvrage qui permet au spectateur de déambuler dans cette multitude de visages et de corps divers qui forment l'Allemagne d'alors. Pour autant, Döblin y insiste dans son texte, il ne s'agit pas de créer de nouveaux stéréotypes, simplement plus raffinés que ceux auxquels ils prétendent se substituer. Le montage de ces images doit aussi permettre au spectateur d'engager un travail comparatif et qui devrait l'entraîner dans une démarche centripète :

Tout comme il existe une anatomie comparée grâce à laquelle on peut d'abord accéder à une conception de la nature et de l'histoire des organes, ce photographe a pratiqué une photographie comparée et a, de la sorte, atteint une position scientifique supérieure aux photographes de détail. Nous sommes libres de lire une foule de choses dans ces images. Ces images composent un matériau réfléchissant l'histoire culturelle, des classes et de l'économie de ces trente dernières années. [...] Face à bon nombre de ces images, on devrait raconter des histoires complètes. Elles y invitent le spectateur, elles constituent un matériau pour les auteurs, un matériau plus stimulant et qui offre davantage que bon nombre de notes de journaux. Voilà mes instructions. Celui qui regarde apprendra vite - mieux que par les exposés et les théories - grâce à ces images claires et puissantes. Et il apprendra quelque chose sur lui-même et sur les autres ${ }^{3}$.

${ }^{1}$ Alfred Döblin, «Von Gesichtern, Bildern und ihrer Wahrheit», dans Manfred Heiting (éd.), August Sander, Cologne, Taschen, 1999. Paru d'abord dans : August Sander, Antlitz der Zeit. Sechzig Aufnahmen deutscher Menschen des 20. Jahrhunderts, op. cit.

${ }^{2}$ Alfred Döblin, « Von Gesichtern, Bildern und ihrer Wahrheit », art. cit., p. 14 (nous traduisons).

${ }^{3}$ Ibid., p. 15 (nous traduisons). 
Si Döblin critique au passage des «notes de journaux » (Zeitungsnotizen) dans lesquelles on pourrait reconnaître Les Employés comme d'autres écrits de Kracauer datant de la même époque, on ne peut s'empêcher de retrouver dans le projet de Sander vu par Döblin, une réalisation concrète d'un montage qui donnerait un aperçu d'une époque (que Döblin décrit encore comme celle d'un bouleversement très rapide de la société) tout en recomplexifiant l'image que s'en faisaient ses contemporains bourgeois jusqu'alors.

Reste la question du réel, de la résistance du réel, qui n'est pas anodine dans le cas de Sander étant donné que le photographe prône et applique, à première vue, une «neutralité » totale du dispositif lors de ses prises de vues. Sander lui-même insiste par exemple sur l'importance d'une photographie qu'il nomme « exacte ${ }^{1}$ et dont l'exactitude peut mener au portrait partiel de la société de son temps. Cette exactitude passe notamment par un dispositif et une mise en scène simples qui consistent à photographier le sujet de face la plupart du temps, en plein pied ou presque, regard caméra, etc. Nombre de commentateurs de l'œuvre de Sander ont aussi souligné l'extraordinaire netteté de la plupart de ces clichés, que le photographe obtient en travaillant avec une chambre dont la cible image est relativement grande. Et à cet égard, Sander apparaît effectivement comme un précurseur du «style documentaire », tant son œuvre réintroduit une forme de limpidité visuelle dans un monde perçu par ses prédécesseurs photographes comme un amoncellement désordonné et devenu illisible ${ }^{2}$.

Pourtant, à bien y regarder, plusieurs images résistent discrètement à cette classification esthétique et historiographique. Dans un portrait sur deux environ, il est possible de déceler plusieurs zones floues. Certes, ces zones sont courantes dans les portraits des années 1920, 1930 et 1940. À l'époque, le travail à la chambre optique pouvait entraîner un léger non-parallélisme entre la cible image et l'objectif - ce qui provoquait une variation de la distance entre les différentes zones d'un même foyer image et la plaque photosensible. Mais ces explications ne tiennent pas dans le cas de Sander. Car ces zones apparaissent non seulement dans des photographies qui frappent par leur piqué général, mais en outre, d'une zone à l'autre, il n'y a pas de cohérence de "plan de netteté ». Les endroits flous de ces portraits ne sont donc pas attribuables techniquement au seul moment de la prise de vue.

\footnotetext{
${ }^{1}$ Hans Gotthard Vierhuff, Die Neue Sachlichkeit. Malerei und Fotografie, Cologne, Dumont, 1980, p. 71.

${ }^{2}$ Voir notamment l'étude très fouillée d'Olivier Lugon, Le Style documentaire. D’August Sander à Walker Evans, 1920-1945, Paris, Éditions Macula, 2001.
} 
Soit ils sont le fait d'une manipulation lors du tirage des épreuves. Dans ce cas, seule l'intention du photographe peut expliquer le brouillage de certaines parties des portraits. Soit nous devons les accepter, sans autre forme de procès, comme des endroits dans lesquels l'image résiste. Impossible de dire si cette apparente résistance appartient au réel photographié (un flou de bougé pour une part infime et a priori non autonome d'un corps ou d'un paysage par ailleurs parfaitement immobile), au dispositif qui a permis de le fixer, ou à l'intention du photographe qui, par une manipulation fine, est parvenu à réintroduire des zones floues dans l'image lors du tirage. Mais quoi qu'il en soit, ces zones apparaissent comme des disfonctionnements qui sont révélés, a contrario, par l'image nette et «neutre» de Sander. Et c'est cette idée que l'on veut en retenir: parce qu'il se met au service d'une démarche précise et maîtrisée par le photographe, le dispositif autorise en contre-point l'identification d'éléments qui, apparemment, lui résistent. Car c'est bien parce que Sander met son art au service d'une absolue netteté qui doit rendre le réel «tel qu'il est », que des parties moins nettes se montrent au spectateur. Autrement dit, l'exactitude du dispositif et son apparente neutralité permettent l'émergence de traces de sa négation, des résistances.

\section{Vers un montage-résistance?}

Selon Kracauer, le plan de cinéma dispose des mêmes potentialités que l'image photographique en ce qu'il peut trahir la mise en scène et le montage lissant. Mais le montage peut-il à son tour être tout à la fois le vecteur (de fait) d'un lissage et, en tant que tel, lieu d'une critique de ce lissage par production/révélation de résistances? Un montage peut-il pointer ces moments dans lesquels quelque chose résiste au lissage cinématographique ? Bien évidemment, ces questions nous éloignent quelque peu des préoccupations de Kracauer pour lequel, comme on l'a dit, le réel ne donne l'impression de «résister » que lorsqu'il est recouvert abusivement par une construction qui s'oppose à sa nature. Néanmoins, un cinéma dont Kracauer aura à peine le temps d'entrevoir les premières manifestations fameuses avant sa mort en 1966, a de toute évidence tenté de réaliser un tel montage. Le Nouveau Cinéma Allemand, à travers les premières œuvres et les théories de son mentor Alexander Kluge (un «montage des associations » non directif qui fait de chaque spectateur pris individuellement un « coauteur du film»), a érigé en programme la volonté de montrer un réel qui résiste à l'intérieur même d'un montage qui ne doit pas l'annihiler. Les multiples contestations du montage classique que les premiers films de Kluge (Anita G. en 1966 ; 
Les Artistes sous le chapiteau: perplexes en 1968 ; Travaux occasionnels d'une esclave en 1973) mettent en pratique par le faux raccord, la confusion entre diégèse et réalité du tournage, ou encore par le montage d'éléments hétérogènes (sans pour autant se faire directif comme chez Eisenstein), réalisent ce désir d'un art mis au service d'une restauration de la complexité du réel et de ses multiples résistances à tout programme imposé. Le noyau de cette restauration est ici encore un regard nécessairement subjectif que l'auteur (le photographe pour Kracauer) doit poser sur le monde pour le sentir, l'investir tout entier, et pour en rendre une part de complexité. Mais il ne peut plus être dissocié d'une seconde subjectivité, fondamentalement multiple cette fois : celle de tous les spectateurs pris individuellement auxquels l'auteur accepte de s'allier dans l'espace du film.

$\mathrm{Au}$ terme provisoire de notre réflexion et de notre tentative de cerner une conception recomposée d'un «bon montage », on s'adosse implicitement à une définition négative du pouvoir du cinéma. Car si les premiers films d'Alexander Kluge par exemple déconstruisent le montage pour rendre au réel sa nature rugueuse et fortuite, ce dé-montage est subordonné à un travail de déconstruction d'une habitude narrative qui colle littéralement aux yeux du spectateur du cinéma classique. Ce sont par conséquent avant tout des films «en opposition », qui offrent au cinéma la possibilité de révéler les résistances du réel sans pour autant lui faire violence. Si nous essayons malgré tout d'imaginer un "bon montage » qui formulerait cette idée de façon positive, on butte sur une impossibilité : celle d'un montage qui révélerait tout à la fois la complexité du réel sans pour autant insister sur sa différence avec le montage qui lui fait violence. Il s'agirait en somme d'un assemblage qui travaillerait la juxtaposition d'éléments sans que cette juxtaposition ne rende des comptes (même par opposition) à un assemblage linéaire et signifiant. Une réalisation positive de cet hypothétique «bon montage » est peut-être à trouver dans quelques expériences cinématographiques et vidéographiques que Kracauer n'aura pas le temps de connaître et qui émergent à partir des années 1960, notamment dans des dispositifs à écrans multiples. Car ces dispositifs contiennent, dans leurs conditions matérielles mêmes, la perpétuelle reconfiguration que réalisait potentiellement le lecteur déambulateur d'un ouvrage de compilation comme Les Employés ${ }^{1}$.

${ }^{1}$ C'est en substance l'hypothèse de départ que nous mettons à l'épreuve de quelques installations récentes dans un second texte à paraître : Maud Hagelstein \& Jeremy Hamers, «L'Atlas à l'épreuve de l'image en mouvement (Warburg, Farocki, Didi- 
Huberman) », dans Mathilde Bert, Ingrid Falque, Maud Hagelstein, Images fixes / Images en mouvement, MethIS, Presses de l'Université de Liège, à paraître, 2016.

227

Bull. anal. phén. XII 4 (2016)

http://popups.ulg.ac.be/1782-2041/ @ 2016 ULg BAP 